\title{
Validation of a capnodynamic method for measuring Effective Lung Volume in healthy volunteers
}

Tomas Öhman 1,2, Thorir Sigmundsson 1,2, Caroline Hällsjö Sander 1,2, Magnus Hallbäck ${ }^{3}$, Anders Oldner 1,2, Håkan Björne ${ }^{1,2}$, ${ }^{1}$ Department of perioperative medicine and intensive care, Karolinska University Hospital, Solna, Sweden, ${ }^{2}$ Department of Physiology and Pharmacology, Karolinska Institutet, Stockholm, Sweden, ${ }^{3}$ Maquet Critical Care AB, Solna, Sweden

\section{Conclusion}

In healthy subjects in the up-right position ELV and FRC showed a high agreement. This indicates that ELV could be used as a continuous online measurement of FRC during mechanical ventilation. Further, ongoing, studies will evaluate the performance of ELV on anaesthetized patients

\section{Background}

Effective lung volume (ELV) can be calculated continuously using a capnodynamic method utilizing a breathing pattern with intermittent cyclic prolongation of inspiratory pauses and correlates well with functional residual capacity (FRC) in healthy lungs in a porcine model.

\section{Aims}

The aim of this study was to evaluate and validate ELV as a volume correlated to FRC in healthy volunteers.

\section{Methods}

10 healthy volunteers were passively ventilated in an upright position using the capnodynamic methods breathing pattern without PEEP. ELV was measured in at least three consecutive minutes where breaths were showing a good fit (low error) between measured and calculated expired CO2. Mean ELV was then compared to $\mathrm{FRC}$ using body box plethysmography $\left(\mathrm{FRC}_{\mathrm{bp}}\right)$, In four out of ten study subjects we also measured FRC by a nitrogen wash out method for measuring FRC in spontaneous breathing subjects (Exhalyzer), (FRCex).

$$
E L V \cdot\left(F_{A} C O_{2}^{n}-F_{A} C O_{2}^{n-1}\right)=E P B F \cdot \Delta t^{n} \cdot\left(C_{v} C O_{2}-C_{c} C O_{2}^{n}\right)-V T C O_{2}^{n}
$$

\section{Results}

Three of the study subjects were excluded since they could not tolerate to be ventilated in the controlled ventilator mode.

ELV showed a FRC (median (range)) of $3350 \mathrm{ml}(2700-4500 \mathrm{ml}) . \mathrm{FRC}_{\mathrm{bp}}$ in the same objects were $3700 \mathrm{ml}(2600-4400$ $\mathrm{ml}) . \mathrm{FRC}_{\mathrm{ex}}$ was slightly higher $3950 \mathrm{ml}(2900-5000 \mathrm{ml})$. ELV and FRC showed high agreement when compared in a Blant-Altman plot with a bias (95\% limits of agreement of) of 21 (-754 to $796 \mathrm{ml}) \mathrm{ml}$ and a percentage error of $22 \%$. ELV correlated well with predicted body weight (PBW), $r=0,75$.

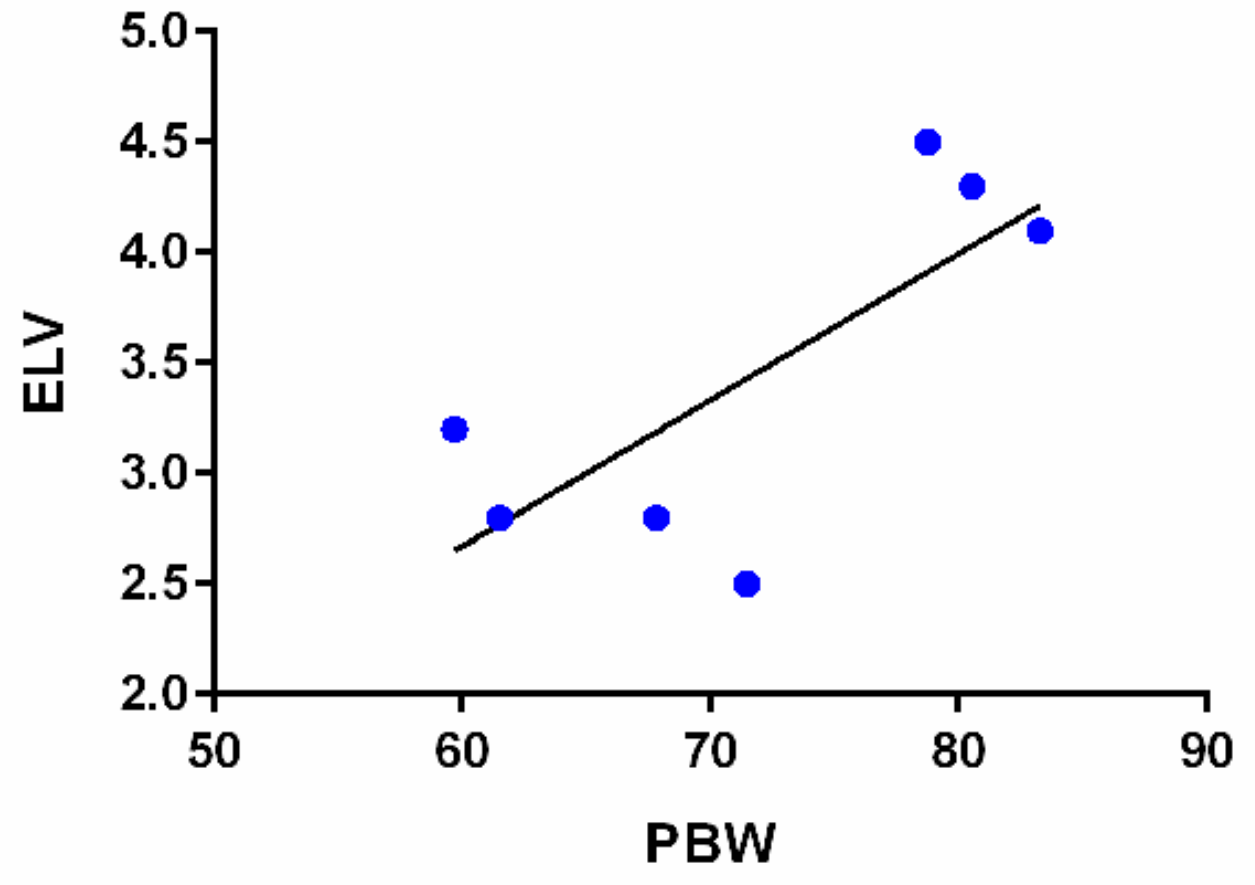

$\mathrm{N}=7$ : Corellation between PBW and ELV

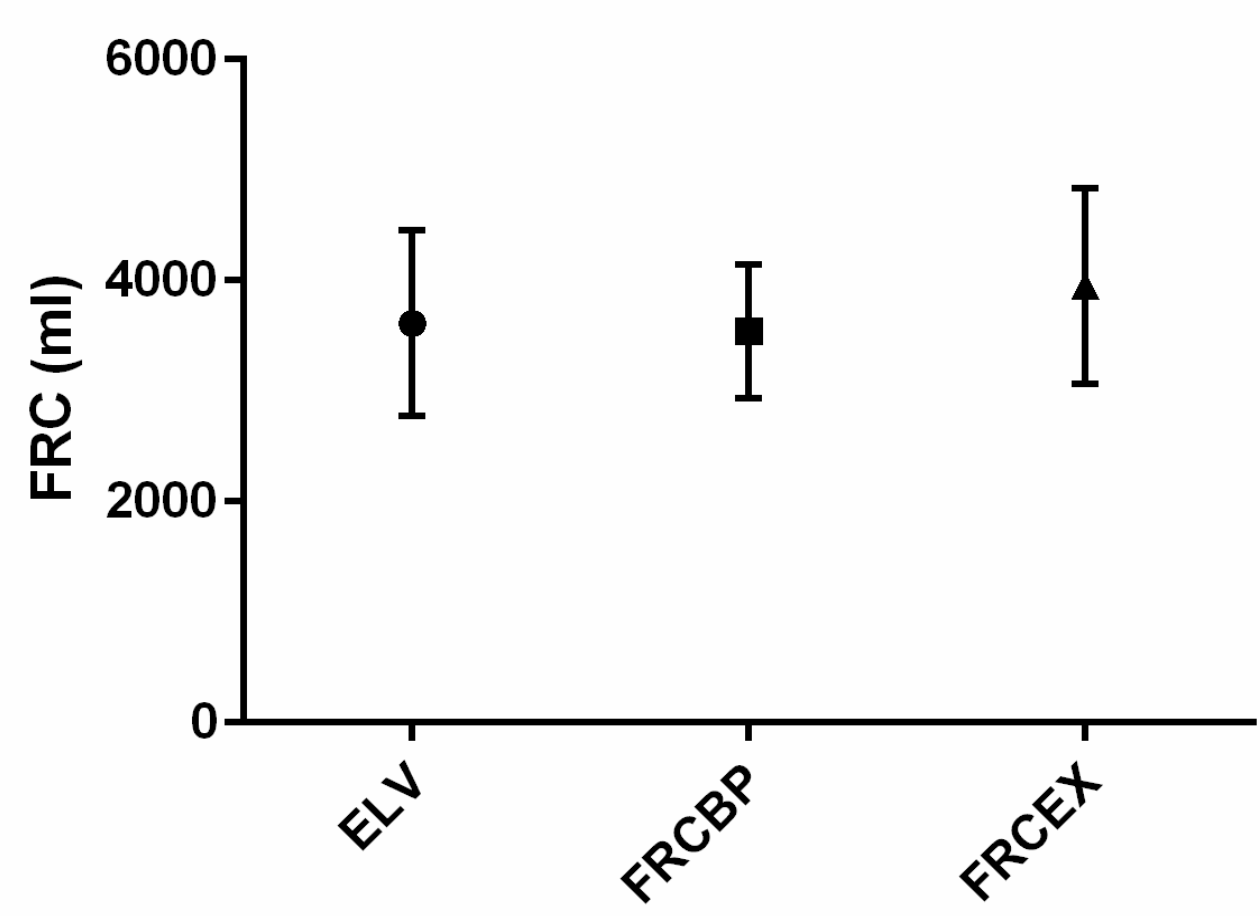

$\mathrm{N}=7$ : Median, range for ELV and FRC 